\title{
Extension of $\lambda-\mu$ B-Spline Curves and Its Application in Surface Modelling
}

\author{
Reenu Sharma \\ Assistant Professor, Department of Mathematics, Mata Gujri Mahila Mahavidyalaya \\ Jabalpur, M.P., India \\ Email: reenusharma6@rediff.com
}

\begin{abstract}
An extension of $\lambda-\mu$ B-spline curves with a shape parameter is presented in this paper. Each curve segmet is generated by four consecutive control points. These curves possess most optimal properties of B-spline basis. We can easily obtain different shapes by altering the value of shape parameter. The lower degree of the basis functions provides less computational complexity. The associated $\lambda$ $\mu B$-spline surfaces can exactly represent the surfaces of revolution.
\end{abstract}

Keywords: $\lambda-\mu$ B-spline curve, shape parameter, blending function, $\lambda-\mu$ B-spline surface, Rotational surface.

\section{Introduction}

Curve and surface modelling is an important tool in the field of CAGD and computer graphics. As a unified mathematical model with many desirable properties, B-splines are widely applied to the modelling of free-form curves and surfaces. However, there are several limitations of the B-spline model, which restrict its applications. For example, once the knot sequences are specified, the positions of B-spline curves are relatively fixed to their control points. On the other hand, when using B-splines to construct interpolation curves, a system of equations must be solved to find the control points. Although the non-uniform rational basis spline (NURBS) can overcome the first shortcoming of B-splines to a certain extent, it fails to model some transcendental curves. The NURBS model has several other potential limitations due to the relative complexity of rational basis functions.

In order to improve the shape of a curve and to overcome the shortcomings of B-splines many bases with shape parameters are presented using trigonometric functions or the blending of polynomial and trigonometric functions in [1-5]. Han presented quadratic trigonometric polynomial curves with one shape parameter [6]. Yan and Liang constructed a class of algebraic-trigonometric blended splines with two parameters $\mathrm{x}$ and $\mathrm{y}$ [7]. Based on quartic blending functions, piecewise polynomial curves with a shape parameter were constructed by Han and Liu [8]. Wang et al. presented piecewise algebraic and trigonometric uniform B-spline curves with a shape parameter of degree $\mathrm{k}(\mathrm{k} \leq 2)$ [9,10].]. Yin and Tan introduced trigonometric polynomial uniform B-splines curves with multiple shape parameters [11]. Extension of uniform cubic B-spline curve with local shape parameters $\lambda_{i}$ are Introduced in [12], in which since only one kind of local shape parameters $\lambda_{i}$ is included, the local adjustability of curves is limited. An algebraic-trigonometric blended piecewise curve with two shape parameters is defined in [13].

A new algorithm for rotational surfaces with two local shape parameters by utilizing the $\lambda-\mu \mathrm{B}$-splines is presented in [14], in which the blending functions are quartic, i.e. of degree 4. So the computational complexities of maintaining the curve increases with increased degree. The higher the degree of a curve, the less controllable it is, in a sense that small changes in coefficients are likely to result in large changes in the shape of the curve. Furthermore, the curves of high degree are more likely to develop bumps in an unpredictable manner when the shape of the curve is changed. On the other hand, lower degree curves have too little flexibility for achieving desired shape in geometric modelling. For these reasons, cubic splines are perfect for obtaining desired shape. The main purpose of this work is to present an extension of $\lambda-\mu$ B-spline curves with a shape parameter in which the blending functions are cubic. The shape of the proposed curve can be adjusted by altering the values of the shape parameter when the control points are fixed. This proposed curve has the same geometric properties as the usual cubic B spline models.

The rest of this paper is organized as follows. In Section 2, the $\lambda-\mu$ B-spline basis functions with two shape parameters are constructed, and some properties of the basis are given. In Section 3, the corresponding $\lambda-\mu$ B-spline curve is defined, the properties of the $\lambda-\mu$ B-spline curve are discussed and effects of the shape parameter on $\lambda-\mu$ B-spline curve are studied. In Section 4 , the corresponding $\lambda-\mu$ Bspline surface with two shape parameter is presented. Conclusion is given in Section 5.

\section{The $\lambda-\mu$ Basis Functions with Two Shape Parameters}

Definition 1. We define the cubic blending functions with two shape parameters $\lambda$ and $\mu$ (which are the extension of [14]) as follows:

$\mathrm{b}_{0,3}(\mathrm{t})=\frac{1}{4}(1-\lambda \mathrm{t})(1-\mathrm{t})^{2}$
$\mathrm{~b}_{1,3}(\mathrm{t})=\frac{1}{2}-\frac{1}{4}(1-\mu(1-\mathrm{t})) \mathrm{t}^{2}$
$\mathrm{~b}_{2,3}(\mathrm{t})=\frac{1}{2}-\frac{1}{4}(1-\lambda \mathrm{t})(1-\mathrm{t})^{2}$
$\mathrm{~b}_{3,3}(\mathrm{t})=\frac{1}{4}(1-\mu(1-\mathrm{t})) \mathrm{t}^{2}$

where $t \in[0,1]$ and $\lambda, \mu \in[-1,1]$. These are called the $\lambda-\mu \mathrm{B}$ spline basis functions with two shape parameters. 


\section{International Journal of Science and Research (IJSR) \\ ISSN (Online): 2319-7064}

Index Copernicus Value (2013): 6.14 | Impact Factor (2015): 6.391

\section{Properties of $\lambda-\mu$ B-spline basis functions}

(a) Terminal Properties: At the endpoints, we know that

$$
\left\{\begin{array} { l } 
{ b _ { 0 , 3 } ( 0 ) = 1 } \\
{ b _ { 3 , 3 } ( 1 ) = 1 }
\end{array} \quad \text { and } \quad \left\{\begin{array}{c}
b_{i, 3}^{(j)}(0)=0 \\
b_{3-i, 3}^{(j)}(1)=0
\end{array}\right.\right.
$$

where $0 \leq \mathrm{j} \leq \mathrm{i}-1 ; \mathrm{i}=1,2,3$ and $\mathrm{b}_{\mathrm{i}, 3}^{(0)}(\mathrm{t})=\mathrm{b}_{\mathrm{i}, 3}(\mathrm{t})$

(b) Non-negativity: The $\lambda-\mu \mathrm{B}$-spline basis are non-negative on the interval $[0,1]$

$$
b_{i, 3}(t) \geq 0, \quad i=0,1,2,3 ; t \in[0,1]
$$

(c) Symmetry: The $\lambda-\mu$ B-spline basis functions are symmetric, namely,

$b_{i, 3}(t)=b_{3-i, 3}(1-t) ; i=0,1,2,3 ; t \in[0,1]$

(d) Partition of unity: The $\lambda$ - $\mu$ B-spline basis possess weight property,

$$
\sum_{i=0}^{3} b_{i, 3}(t)=1 ; t \in[0,1]
$$

(e) Maximum Value: The maximum value of $b_{i, 3}(t)$ occurs at $\mathrm{t}=\frac{\mathrm{i}}{3}, \mathrm{i}=0,1,2,3$.

Figure 1 shows the curves of the $\lambda-\mu \mathrm{B}$-spline basis functions for $\lambda=\mu=-1,0,1$ where $b_{0,3}(t), b_{1,3}(t), b_{2,3}(t)$ and $b_{3,3}(t)$ are represented by blue, red, green and black lines respectively.

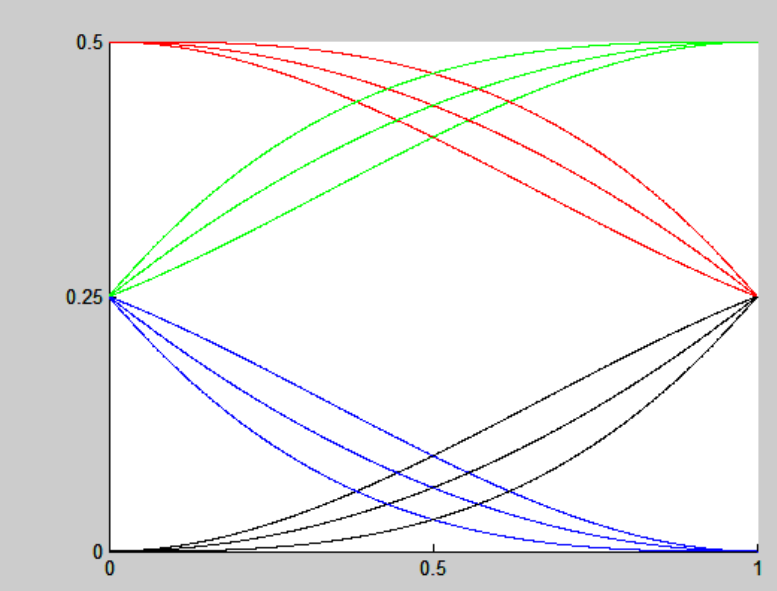

Figure 1: $\lambda-\mu$ B-spline basis functions for $\lambda=\mu=-1,0,1$

\section{The $\lambda-\mu$ B-Spline Curves with Two Shape Parameters}

Definition 2. Given control points $P_{i}(i=0,1, \ldots, n)$ in $R^{2}$ or $\mathrm{R}^{3}$ and a knot vector $\mathrm{U}=\left(\mathrm{u}_{0}, \mathrm{u}_{1}, \ldots, \mathrm{u}_{\mathrm{n}+3}\right)$. Then the curves

$$
\mathrm{T}_{\mathrm{i}, 3}(\lambda, \mu ; \mathrm{t})=\sum_{\mathrm{j}=0}^{3} \mathrm{~b}_{\mathrm{j}, 3}(\mathrm{t}) \mathrm{P}_{\mathrm{i}+\mathrm{j}-3}
$$

for $\mathrm{t} \in[0,1], \mathrm{i}=1,2, \ldots, \mathrm{n}$.

are called cubic $\lambda-\mu$ B-spline curve segments, where $b_{j, 3}(j=$ $0,1,2,3)$ are $\lambda-\mu$ B-spline basis functions. All the curve segments make up the piecewise cubic blending spline curves with parameters $\lambda$ and $\mu$, defined by

$$
\mathrm{T}(\lambda, \mu ; \mathrm{u})=\mathrm{T}_{\mathrm{i}, 3}\left(\lambda, \mu ; \frac{\mathrm{u}-\mathrm{u}_{\mathrm{i}}}{\Delta \mathrm{u}_{\mathrm{i}}}\right)
$$

for $\mathrm{u} \in\left[\mathrm{u}_{\mathrm{i}}, \mathrm{u}_{\mathrm{i}+1}\right] \subset\left[\mathrm{u}_{3}, \mathrm{u}_{\mathrm{n}+1}\right]$, where $\Delta \mathrm{u}_{\mathrm{i}}=\mathrm{u}_{\mathrm{i}+1}-\mathrm{u}_{\mathrm{i}}$ and $\mathrm{i}=3,4, \ldots, \mathrm{n}$. $\mathrm{T}(\lambda, \mu ; \mathrm{u})$ is called $\lambda-\mu \mathrm{B}$-Spline curve with two shape parameters $\lambda$ and $\mu$.

\section{Properties of $\lambda$ - $\mu$ B-spline curves}

The $\lambda$ - $\mu$ B-Spline curves (2) have the following properties:

(a) Terminal Properties:

$$
\begin{aligned}
& \mathrm{T}(\lambda, \mu ; 0)=\frac{1}{4}\left(\mathrm{P}_{0}+2 \mathrm{P}_{1}+\mathrm{P}_{2}\right) \\
& \mathrm{T}(\lambda, \mu ; 1)=\frac{1}{4}\left(\mathrm{P}_{1}+2 \mathrm{P}_{2}+\mathrm{P}_{3}\right) \\
& \mathrm{T}^{\prime}(\lambda, \mu ; 0)=\left(\mathrm{P}_{2}-\mathrm{P}_{0}\right)\left(\frac{\lambda+2}{4}\right) \\
& \mathrm{T}^{\prime}(\lambda, \mu ; 1)=\left(\mathrm{P}_{3}-\mathrm{P}_{1}\right)\left(\frac{\mu+2}{4}\right) \\
& \mathrm{T}^{\prime \prime}(\lambda, \mu ; 0)=\left(\mathrm{P}_{0}-\mathrm{P}_{2}\right)\left(\frac{2 \lambda+1}{2}\right)+\left(\mathrm{P}_{1}-\mathrm{P}_{3}\right)\left(\frac{\mu-1}{2}\right) \\
& \mathrm{T}^{\prime \prime}(\lambda, \mu ; 1)=\left(\mathrm{P}_{3}-\mathrm{P}_{1}\right)\left(\frac{2 \mu+1}{2}\right)+\left(\mathrm{P}_{2}-\mathrm{P}_{0}\right)\left(\frac{\lambda-1}{2}\right)
\end{aligned}
$$

(b) Symmetry:

$\mathrm{P}_{0}, \mathrm{P}_{1}, \mathrm{P}_{2}, \mathrm{P}_{3}$ and $\mathrm{P}_{3}, \mathrm{P}_{2}, \mathrm{P}_{1}, \mathrm{P}_{0}$ define the same $\lambda$ - $\mu$ B-spline curve in different parametrizations, i.e.,

$\mathrm{T}\left(\lambda, \mu ; \mathrm{t} ; \mathrm{P}_{0}, \mathrm{P}_{1}, \mathrm{P}_{2}, \mathrm{P}_{3}\right)=\mathrm{T}\left(\mu, \lambda ; 1-\mathrm{t} ; \mathrm{P}_{3}, \mathrm{P}_{2}, \mathrm{P}_{1}, \mathrm{P}_{0}\right)$

for $t \in[0,1], \lambda, \mu \in[-1,1]$.

(c) Geometric invariance:

The shape of a $\lambda-\mu \mathrm{B}$-spline curve is independent of the choice of coordinates, i.e. (2) satisfies the following two equations:

$\mathrm{T}\left(\lambda, \mu ; \mathrm{t} ; \mathrm{P}_{0}+\mathrm{q}, \mathrm{P}_{1}+\mathrm{q}, \mathrm{P}_{2}+\mathrm{q}, \mathrm{P}_{3}+\mathrm{q}\right)=$

$\mathrm{T}\left(\lambda, \mu ; \mathrm{t} ; \mathrm{P}_{0}, \mathrm{P}_{1}, \mathrm{P}_{2}, \mathrm{P}_{3}\right)+\mathrm{q}$

$\mathrm{T}\left(\lambda, \mu ; \mathrm{t} ; \mathrm{P}_{0} * \mathrm{M}, \mathrm{P}_{1} * \mathrm{M}, \mathrm{P}_{2} * \mathrm{M}, \mathrm{P}_{3} * \mathrm{M}\right)=$

$\mathrm{T}\left(\lambda, \mu ; \mathrm{t} ; \mathrm{P}_{0}, \mathrm{P}_{1}, \mathrm{P}_{2}, \mathrm{P}_{3}\right) * \mathrm{M}$

for $t \in[0,1], \lambda, \mu \in[-1,1]$

where $\mathrm{q}$ is arbitrary vector in $\mathrm{R}^{2}$ or $\mathrm{R}^{3}$, and $\mathrm{M}$ is an arbitrary $\mathrm{d} \times \mathrm{d}$ matrix, $\mathrm{d}=2$ or 3 .

(d) Convex hull property:

Since the $\lambda-\mu$ B-spline basis functions for $\lambda=\mu=-1,0,1$ have the properties of non-negativity and partition of unity, the entire $\lambda-\mu \mathrm{B}$-spline curve segment must lie inside the control polygon spanned by $\mathrm{P}_{\mathrm{i}-3}, \mathrm{P}_{\mathrm{i}-2}, \mathrm{P}_{\mathrm{i}-1}, \mathrm{P}_{\mathrm{i}}$.

\section{Shape Analysis of the $\lambda$ - $\mu$ B-Spline Curves}

Given a control polygon, the shape of the usual cubic Bspline curve is fixed but for a cubic $\lambda-\mu$ B-spline curve the $^{\text {the }}$ shape can be changed by altering the values of shape parameters $\lambda$ and $\mu$.

For $t \in[0,1]$, we rewrite (2) as follows:

$\mathrm{T}_{\mathrm{i}, 3}(\lambda, \mu ; \mathrm{t})=\sum_{\mathrm{j}=0}^{3} \mathrm{c}_{\mathrm{j}, 3}(\mathrm{t}) \mathrm{P}_{\mathrm{i}+\mathrm{j}-3}+\frac{\lambda \mathrm{t}}{4}\left(1+\mathrm{t}^{2}-2 \mathrm{t}\right)\left(\mathrm{P}_{\mathrm{i}-1}-\right.$ $\mathrm{Pi}-3+\mu \mathrm{t} 24(\mathrm{t}-1) \mathrm{Pi}-\mathrm{Pi}-2$

where $c_{0,3}(t)=\frac{1}{4}\left(1+t^{2}-2 t\right), c_{1,3}(t)=\left(2-t^{2}\right), c_{2,3}(t)=$ $\frac{1}{4}\left(1-\mathrm{t}^{2}+2 \mathrm{t}\right), \mathrm{c}_{3,3}(\mathrm{t})=\frac{\mathrm{t}^{2}}{4}, \mathrm{t} \in[0,1], \lambda, \mu \in[-1,1]$

From (4) we can predict the following behaviour of the curve:

i. When we select the same $\lambda$ but different $\mu$, the cubic $\lambda$ $\mu$ B-spline curve segment $T_{i, 3}(\lambda, \mu ; t$ ) approaches to (or 


\section{International Journal of Science and Research (IJSR) \\ ISSN (Online): 2319-7064}

Index Copernicus Value (2013): 6.14 | Impact Factor (2015): 6.391

departs from) the line segment $\mathrm{P}_{\mathrm{i}-3} \mathrm{P}_{\mathrm{i}-1}$ with the increasing (or decreasing) of shape parameters $\mu$.

ii. When we select the same $\mu$ but different $\lambda$, the cubic $\lambda-\mu$ B-spline curve segment $\mathrm{T}_{\mathrm{i}, 3}(\lambda, \mu ; \mathrm{t}$ ) approaches to (or departs from) the line segment $\mathrm{P}_{\mathrm{i}-2} \mathrm{P}_{\mathrm{i}}$ with the increasing (or decreasing) of shape parameters $\lambda$.

iii. When we select different $\lambda$ and $\mu$ the cubic $\lambda-\mu$ Bspline curve segment $\mathrm{T}_{\mathrm{i}, 3}(\lambda, \mu ; \mathrm{t}$ ) approaches to (or departs to) overall the control polygon with the concurrent increasing (or decreasing) of shape parameters $\lambda$ and $\mu$.

Figure 2 shows a computed example of cubic $\lambda$ - $\mu$ B-spline curve with different values of shape parameter $\lambda$ and fixed value of $\mu$. These curves are shown by blue lines which are

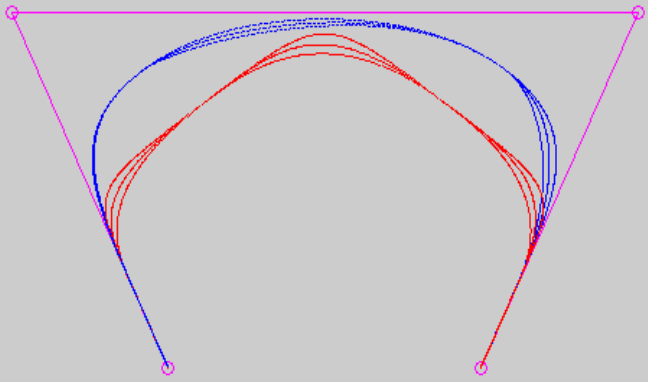

Figure 2: Cubic $\lambda$ - $\mu$ B-spline curve with different values of $\lambda$ and fixed $\mu$.

Figure 3: Cubic $\lambda-\mu$ B-spline curve with different values of $\mu$ and fixed $\lambda$.

generated by setting $\lambda=-1,0,1)$ and $\mu=1$. Similarly figure 3 shows a computed example of cubic $\lambda-\mu$ B-spline curve with different values of shape parameter $\mu$ and fixed value of $\lambda$. These curves are shown by blue lines which are generated by setting $\mu=-1,0,1)$ and $\lambda=1$. The corresponding cubic $\lambda$ $\mu$ B-spline curve of [14] is shown by red lines in both figure 2 and figure 3 . Thus the cubic $\lambda-\mu \mathrm{B}$-spline curve can be made closer to the control polygon than the classical cubic
B-spline curve with Bernstein basis. Hence the shape parameter $\lambda$, $\mu$ provides an efficient tool for obtaining various smooth shapes in geometric modelling.

\section{The $\lambda-\mu$ B-Spline Surfaces}

Given control points $P_{i j}(i=0,1, \ldots, n ; j=0,1, \ldots, m)$ and the knot vectors $U=\left(\mathrm{u}_{0}, \mathrm{u}_{1}, \mathrm{u}_{2}, \ldots, \mathrm{u}_{\mathrm{i}}, \ldots, \mathrm{u}_{\mathrm{n}+3}\right)$ and $\mathrm{V}=$ $\left(v_{0}, v_{1}, v_{2}, \ldots, v_{j}, \ldots, v_{m+3}\right)$, using the tensor product method, we can construct cubic $\lambda$ - $\mu$ B-spline surface pieces

$$
\mathrm{T}_{\mathrm{j}, 3}(\mathrm{u}, \mathrm{v})=\sum_{\mathrm{k}=0}^{3} \sum_{\mathrm{l}=0}^{3} \mathrm{~b}_{\mathrm{k}, 3}\left(\lambda_{1} \mu_{1} ; \mathrm{u}\right) \mathrm{b}_{\mathrm{l}, 3}\left(\lambda_{2} \mu_{2} ; \mathrm{v}\right) \mathrm{P}_{\mathrm{i}+\mathrm{k}-3} \mathrm{P}_{\mathrm{j}+\mathrm{l}-3}
$$

where $b_{k, 3}\left(\lambda_{1} \mu_{1} ; u\right)$ and $b_{1,3}\left(\lambda_{2} \mu_{2} ; v\right)$ are the cubic $\lambda-\mu \mathrm{B}$ spline basis functions with shape parameters $\lambda_{1}, \mu_{1}$ and $\lambda_{2}, \mu_{2}$ respectively. These surface pieces constitute cubic $\lambda-\mu \mathrm{B}$ spline surface

$\mathrm{T}\left(\lambda_{1} \mu_{1} ; \lambda_{2} \mu_{2} ; \mathrm{u}, \mathrm{v}\right)$

$$
=\mathrm{T}_{\mathrm{j}, 3}\left(\lambda_{1} \mu_{1} ; \frac{\mathrm{u}-\mathrm{u}_{\mathrm{i}}}{\Delta \mathrm{u}_{\mathrm{i}}}, \lambda_{2} \mu_{2} ; \frac{\mathrm{v}-\mathrm{v}_{\mathrm{j}}}{\Delta \mathrm{v}_{\mathrm{j}}}\right)
$$

Where $u \in\left[\mathrm{u}_{\mathrm{i}}, \mathrm{u}_{\mathrm{i}+1}\right] \quad \subset\left[\mathrm{u}_{3}, \mathrm{u}_{\mathrm{n}+1}\right], \quad \mathrm{v} \in\left[\mathrm{v}_{\mathrm{i}}, \mathrm{v}_{\mathrm{i}+1}\right] \subset$ $\left[\mathrm{v}_{3}, \mathrm{v}_{\mathrm{n}+1}\right], \Delta \mathrm{u}_{\mathrm{i}}=\mathrm{u}_{\mathrm{i}+1}-\mathrm{u}_{\mathrm{i}}, \quad \Delta \mathrm{v}_{\mathrm{i}}=\mathrm{v}_{\mathrm{i}+1}-\mathrm{v}_{\mathrm{i}}, \quad \mathrm{i}=3,4, \ldots, \mathrm{m}$, $\mathrm{j}=3,4, \ldots, \mathrm{n}$. Equation (5) is called cubic $\lambda-\mu$ B-spline surface.

These surfaces have properties similar to the corresponding cubic $\lambda-\mu$ B-spline curves. In addition, since the surfaces have four shape parameters, the shape of the surfaces can be adjusted from two direction (in each direction using two shape parameter), so they can more conveniently be used in the outline design. An example of surface designing by cubic $\lambda-\mu \mathrm{B}$-spline curves is shown in figure 4. 


\section{References}

[1] J. W. Zhang, "C-curves: an extension of cubic curves", Computer Aided Geometric Design, vol. 13, Apr. 1996, pp. 199-217.

[2] J. W. Zhang and Krause F.-L, "Extending cubic uniform B-splines by unified trigonometric and hyperbolic basis". Graphic Models, vol. 67, Mar. 2005, pp. 100-119.

[3] E. Mainar, J. M. Peña, J. Sánchez-Reyes, "Shape preserving alternatives to the rational Bézier model". Computer Aided Geometric Design, vol. 18, Feb. 2001, pp. 37-60.

[4] Y. G. Lü, G. Z. Wang and X. N. Yang, "Uniform trigonometric polynomial B-spline curves", Science in China (Series F), vol. 45, Oct. 2002, pp. 335-343.

[5] G. Z. Wang, Q. Y. Chen and M. H. Zhou, "NUAT Bspline curves", Computer Aided Geometric Design, vol. 21, Feb. 2004, pp. 193-205.

[6] X.L. Han, Quadratic trigonometric polynomial curves with a shape parameter, Comput. Aided Geom. Des., 19 (7) (2002), pp. 503-512.

[7] L.L. Yan, J.F. Liang, A class of algebraictrigonometric blended splines, J. Comput. Appl. Math 235, (6) (2011), pp. 1713-1729.

[8] X.L. Han, S.J. Liu, An extension of the cubic uniform B-spline curves, J. Comput. Aided Des. Comput. Graph., 15 (5), (2003) pp. 576-578.

[9] W.T. Wang, G.Z. Wang, Uniform B-spline with shape parameter, J. Comput. Aided Des. Comput. Graph. 16 (6) (2004), pp. 783-788.

[10] W.T. Wang, G.Z. Wang, Trigonometric polynomial uniform B-spline with parameter, Chin. J. Comput., 28 (7), (2005) pp. 1192-1198.

[11] C.J. Yin, J.Q. Tan, Trigonometric polynomial uniform B-spline curves and surfaces with multiple shape parameters, J. Comput. Aided Des. Comput., Graph., 23 (7), (2011), pp. 1131-1138.

[12] G. Xu, G.Z. Wang, Extension of uniform cubic B-spline curve with local shape parameters, J Comput., Res. Dev, 44 (6), (2007), pp. 1032-1037.

[13] Lanlan Yan, Tao Huang and Rongsheng Wen, "An Algebraic-trigonometric Blended Piecewise Curve", Journal of Information \& Computational Science 12 (17), (2015), pp. 6491-6501.

[14] Gang Hua, Xinqiang Qina, Xiaomin Ji, GuoWeib and Suxia Zhanga, "The construction of $\lambda-\mu \mathrm{B}$-spline curves and its application to rotational surfaces", Applied Mathematics and Computation, 266 (2015), pp.194211. 